Historic, Archive Document

Do not assume content reflects current scientific knowledge, policies, or practices. 

A 58.9

R31

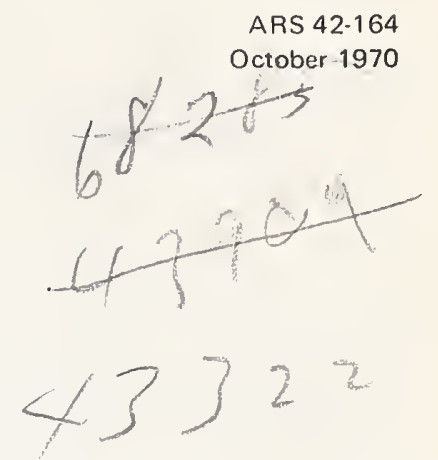

\section{FLIGHT MILL SYSTEM FOR STUDYING INSECT BEHAVIOR}

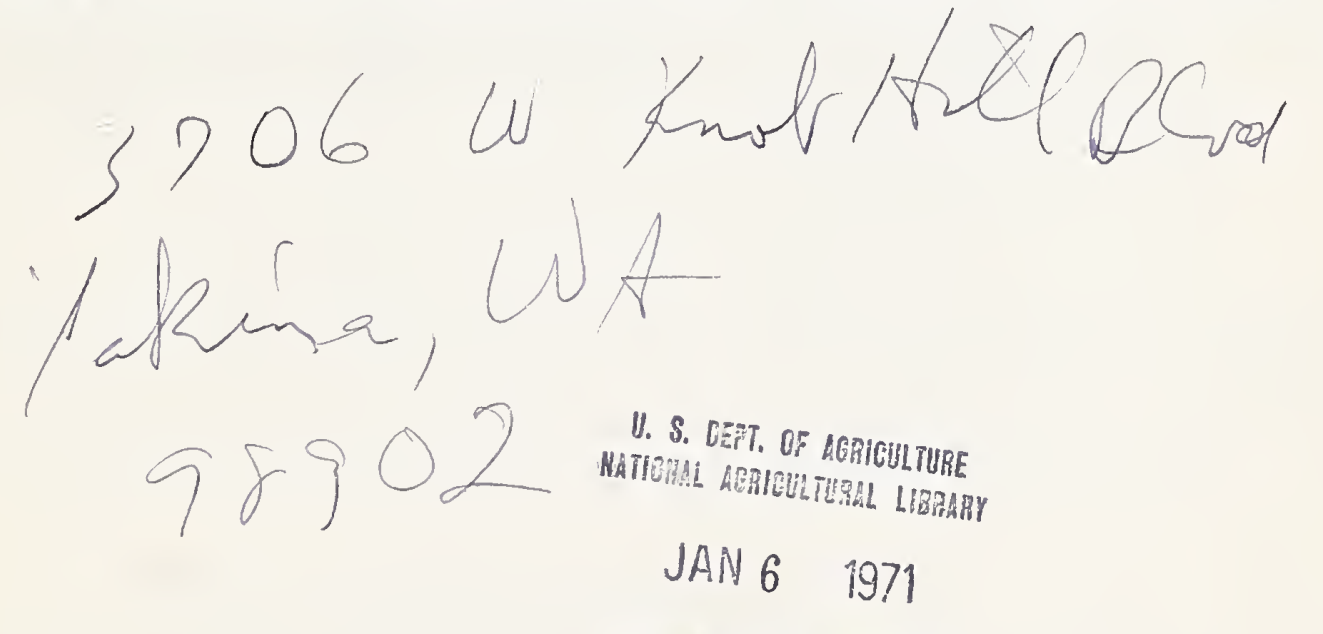

CURBEIT SERIAL RECOROS 
Trade names are used in this publication solely for the purpose of providing specific information. Mention of a trade name does not constitute a guarantee or warranty of the product by the U.S. Department of Agriculture or an endorsement by the Department over other products not mentioned. 


\title{
FLIGHT MILL SYSTEM FOR STUDYING INSECT BEHAVIOR
}

\author{
L. G. Schoenleber, L. D. White, and B. A. Butt ${ }^{1}$
}

A flight mill is an apparatus that confines an insect to a circular flight path when the insect is attached to a pivoted arm. It is a convenient research tool for comparing vigor and flight responses of insects under controlled conditions. Retention of insect vigor is essential for laboratory-reared insects that are sterilized and released to control native populations. ${ }^{2}$ Flight mills can be used to compare the effects of chemosterilants, radiation, diet, rearing practices, and environment on the flight vigor of insects. Flight mills used by Rowley and others, ${ }^{3}$ Kishaba and others, ${ }^{4}$ Smith and Furniss, ${ }^{5}$ and Chambers and O'Connell ${ }^{6}$ have short circular flight paths. For codling moth studies, a flight mill was designed with a larger circular flight path than those used by earlier investigators. The larger diameter flight path reduced the effects of uneven stress on insect wings noted when using mills with smaller flight paths.

\section{FLIGHT MILL DESIGN CHARACTERISTICS}

The flight mill was designed to provide a record of the time of flight, and of revolutions of the flight arm. Records of the specific time of flight, average flight speed, rest periods, total flight period, and distance of flight for the life of each moth tested are provided. The flight mill also performs with minimum friction drag on the flight arms. It can be used to test four or more insects at one time and the insects are easily attached. The large circular flight paths minimize insect lateral fatigue. The flight mill frame is isolated from the recording equipment.

\section{DESIGN AND OPERATION OF FLIGHT MILL}

Two different flight arm systems were developed. One unit uses jewel-magnet bearings mounted on a metal frame (fig. 1). The other unit uses floating magnets on a vertical rod mounted on a base plate (fig. 2). Similar sensors and recording equipment can be used with either flight arm unit to record insect flight activity.

The flight arm system that uses jewel-magnet bearings can be used to compare four codling moths at once. It consists essentially of four flight arms that confine attached insects to circular flight paths. Instruments are also provided to record the time of insect flight and to indicate the revolutions of each flight arm. An electrical cable connects the sensing elements, which are mounted on the main frane of the flight mill, to the instrument panel. Thus the researcher can place the flight mill unit in a controlled environmental chamber and the instruments in a different area. Instruments indicate the flight of each insect during the test without disturbing the insect's activity.

The floating-magnet flight arm unit can also be used to compare two or more moths at one time. Devices to sense the revolutions of each flight arm must be mounted on a separate bracket (not shown in figure 2).

\footnotetext{
${ }^{1}$ Agricultural engineer, Agricultural Enginecring Research Division, and entomologists, Entomology Research Division, respectively, Agricultural Research Service, U.S. Department of Agriculture, Yakima, Wash.

${ }^{2}$ Knipling, E. F. The potential role of the sterility method for insect population control with special reference to combining this method with conventional methods. U.S. Dept. Agr., Agr. Res. Scrv. ARS 33-98. 1964.

${ }^{3}$ Rowley, Wayne A., Graham, C. L., and Williams, R. E. A flight mill system for the laboratory study of mosquito flight. Ent. Soc. Amer. Annals 61(6): 1507-1514. 1968.

${ }^{4}$ Kishaba, A. N., llenncberry, T. J., Hancock, P. F., and Toba, H. H. Laboratory technique for studying flight of cabbage looper moths and the effects of age, sex, food, and tepa on flight characteristics. Jour. Econ. Ent. 60(2): 359-366. 1967.

${ }^{5}$ Smith, 11. W., and liurniss, M. M. An automatically recording insect flight mill. Canad. Ent. 98(3): 249-252. 1966.

${ }^{6}$ Chambers, D. L., and O'Connell, T. B. A flight mill for studies with the Mexican fruit fly. Ent. Soc. Amer. Annals 62(4): $917-920.1969$.
} 


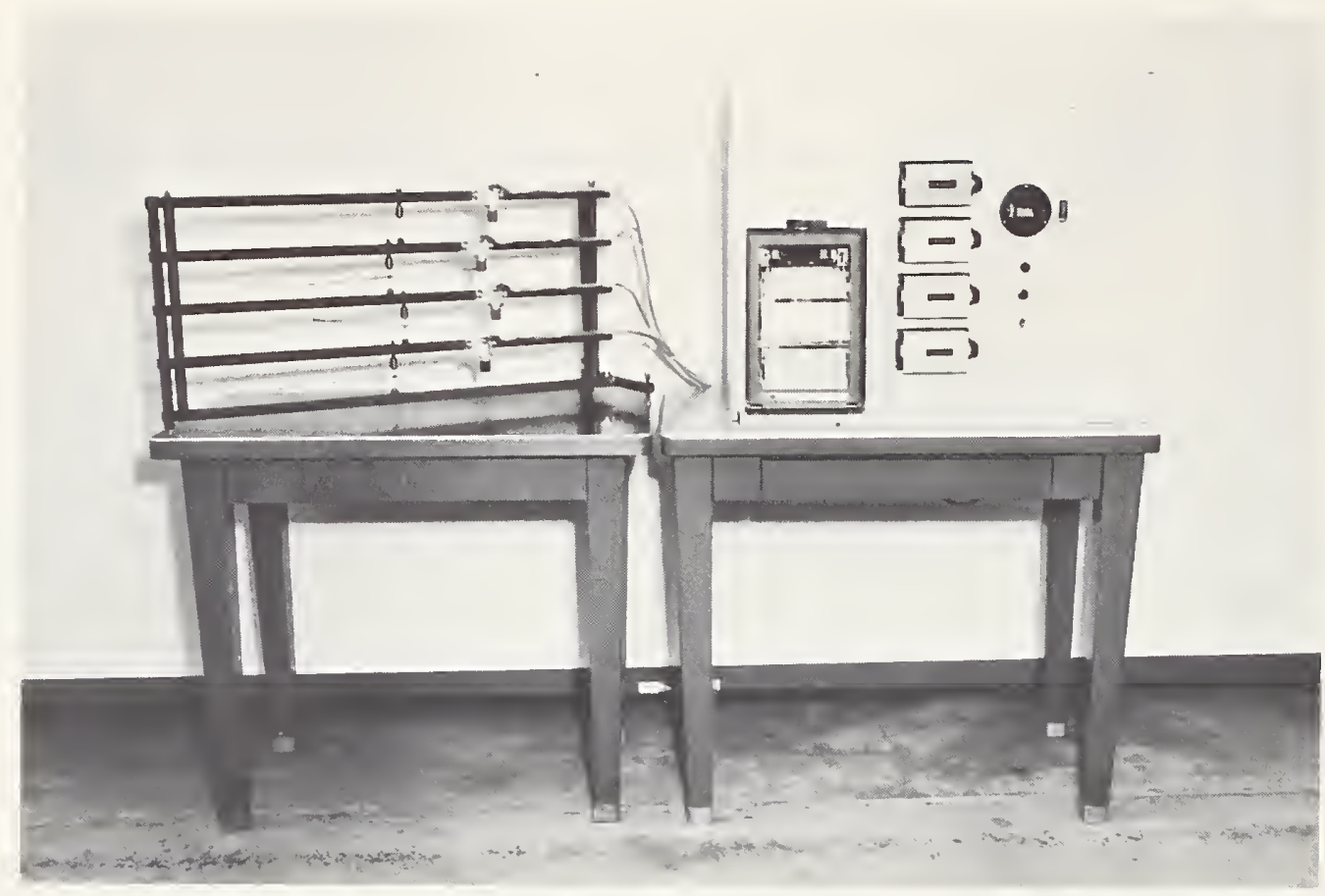

A

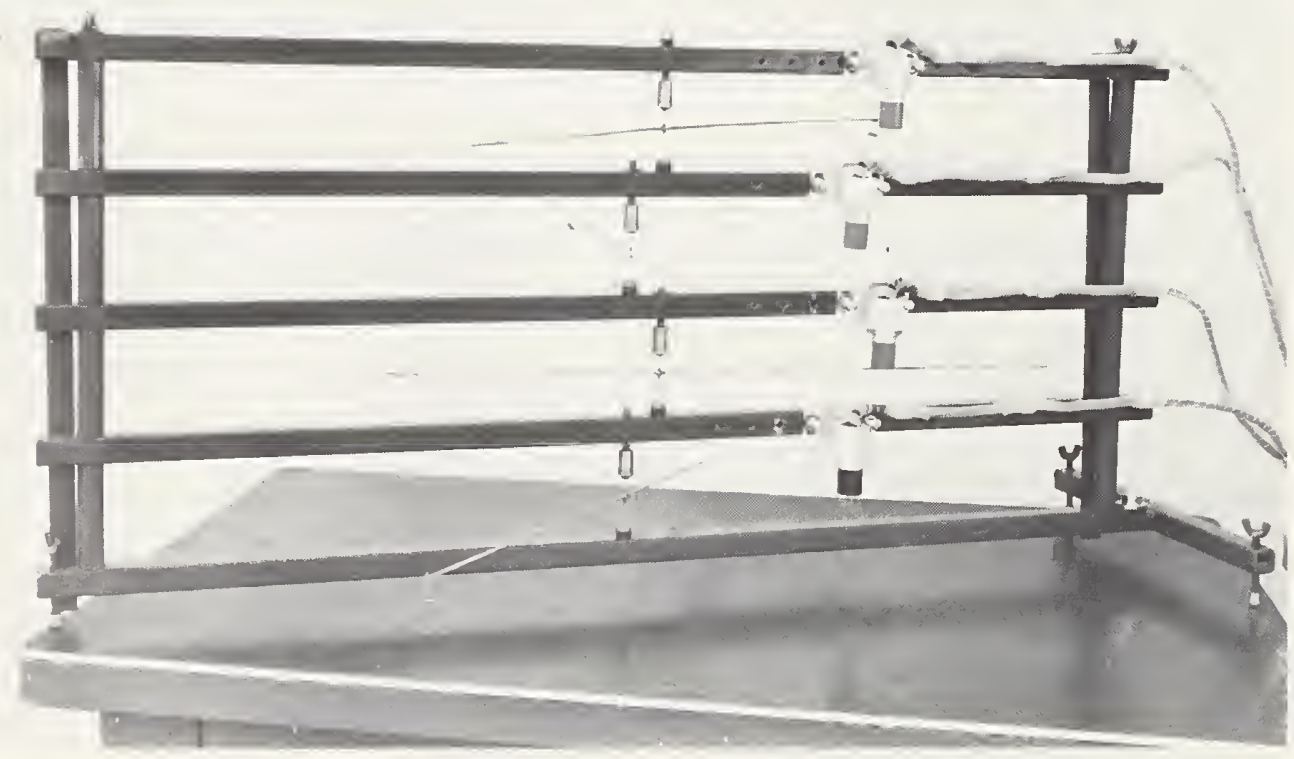

B

Figure 1.- Top, flight mill for four insects, with instruments to record flight activity. Bottom, closeup of flight arm unit. 


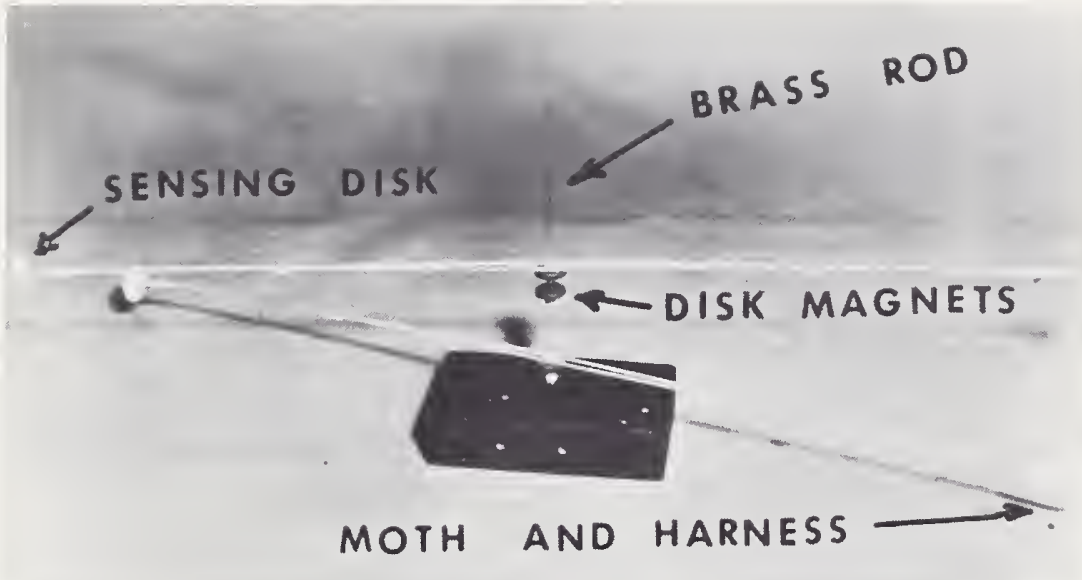

Figure 2.-Floating-magnet flight arm unit.

Light sensing equipment used successfully on earlier tests was replaced with proximity amplifier switches and sensors to eliminate possible influence of light on the insects. The following block diagram shows the components of the flight mill:

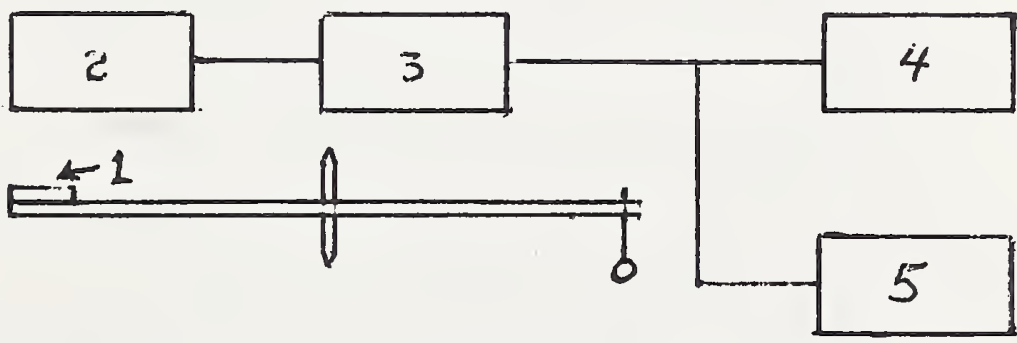

1. Flight arm with disk (aluminum, 1 in. in diameter, 1/64 in. thick).

2. All-metal proximity sensor.

3. Proximity amplifier with relay switch.

4. Digital counter, reset type, continuous duty.

5. Event recorder.

The top bearing consists of a magnet with a threaded rod glued to one end and a steel plug glued to the other end. (fig. 3). The glass jewel mounted in the center of the steel plug acts as a pivot point for the flight arm. The bottom bearing has the same general makeup as the top bearing, but has no threaded rod. The top bearing has a glass jewel, spring-mounted in a No. 3-56 $\mathrm{NF}^{7}$ threaded brass screw. The bottom jewel bearing is cushion-mounted in a No. 3-56 threaded brass screw. The assembled bearings are termed "jewel-magnet bearings."

${ }^{7}$ No. 3-56 NF is an abbreviation for an American National Standard machine screw thread, gage No. $3,0.099$ inch in diameter, with 56 threads per inch, National Fine thread series. 


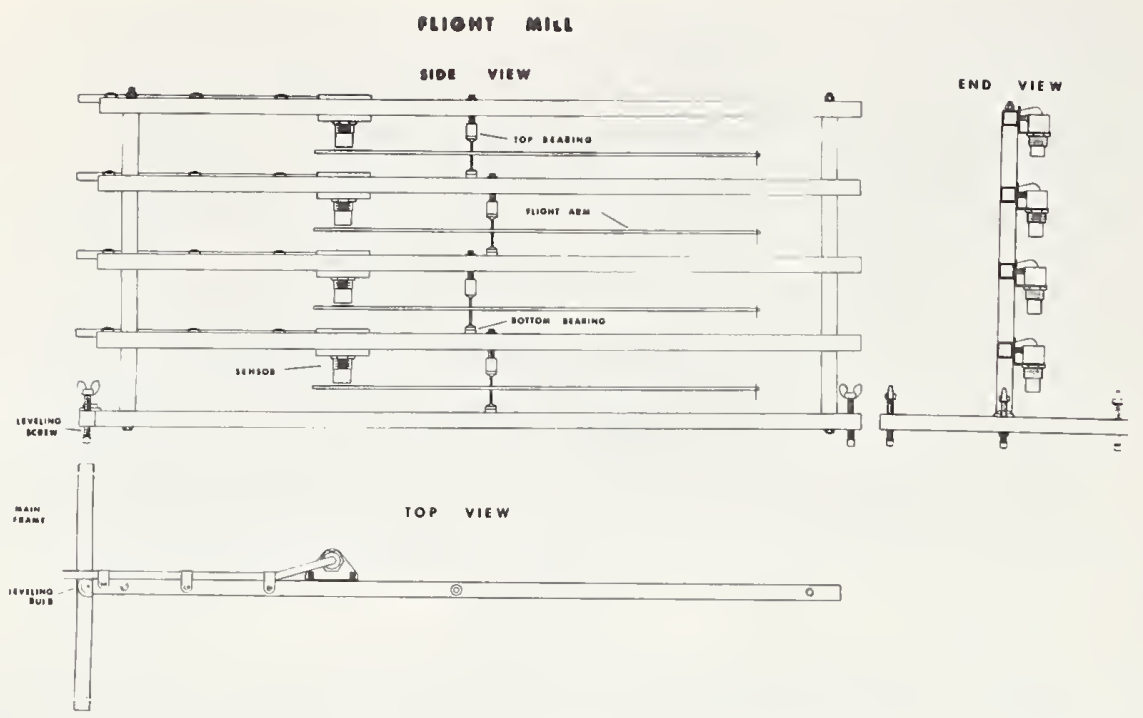

A

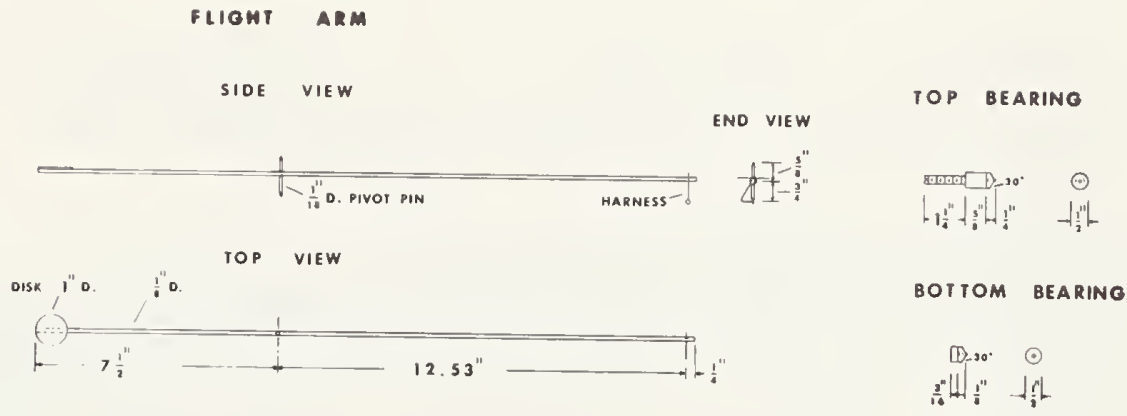

B

Figure 3.-(A) Flight arm unit for flight mill, and (B) flight arm unit and jewel-magnet bearings of flight mill.

The entire flight arm unit consists of balsa wood, 1/8 inch in diameter and $201 / 4$ inches long, a small steel pivot pin, a No. 30 (0.014 inch in diameter) wire harness for attaching the insect, and an aluminum disk, and weighs less than 2 grams (fig. 3). The pivot pin is made of magnetic material. Both ends of the pin are ground and polished to a point with a $60^{\circ}$ angle to fit into the jewel of the bearings. The pivot pin is attached at right angles to the balsa wood through the center of the stick, then glued in position. The distance from the pivot pin to the moth harness is 12.53 inches. The flight arm is balanced with the same amount of weight on each side of the pivot pin. The top jewel-magnet bearing provides sufficient magnetism to hold the pivot arm in a lifted position against the top bearing. The top magnet is Alnico, $1 / 2$ inch in diameter, 5/8 inch long, with $13 / 4$-pounds pull. The bottom magnet is ceramic, $1 / 2$ inch in diameter, $3 / 16$ inch thick, with 1/8-pound pull. The bottom jewel-magnet bearing, which has 7 percent of the magnetic strength of the top magnet, is used to hold the pivot pin in a vertical position. Magnetism holds the magnet of the bottom bearing to the steel frame of the flight mill. The combination jewel-magnet bearings are used to position the flight arm pivot pins and to obtain minimum bearing drag. 
An aluminum foil disk, stiffened with paper backing and attached near the end of the flight arm, actuates the sensor of a proximity amplifier and relay switch as the disk passes near the sensor. Appropriate sensitivity adjustment is provided in the amplifier for any slight variation between the sensor and aluminum disk. Each sensor with proximity amplifier and switch actuates both a digital counter and an analog event recorder. The sensor does not cause any frictional drag on the flight arm.

The harness to hold the adult moth is made of wire 0.014 inch in diameter. It is approximately 2.4 inches long and is bent to one of the shapes shown in fig. 4 .

SIDE

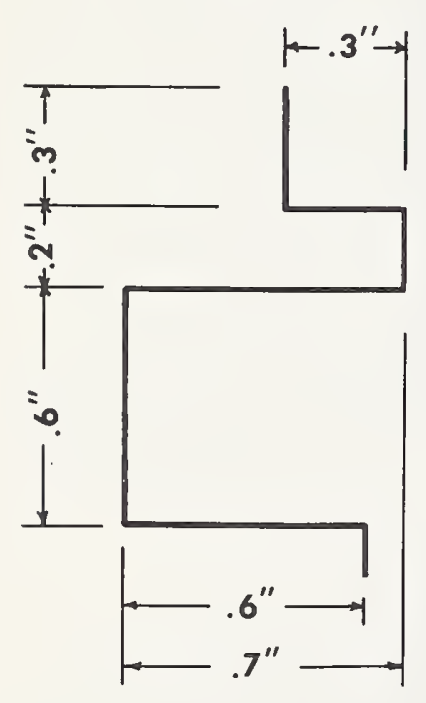

FRON T

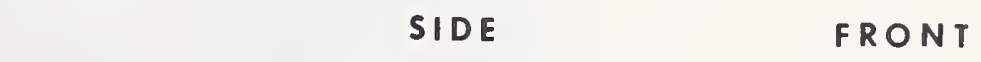

Type A

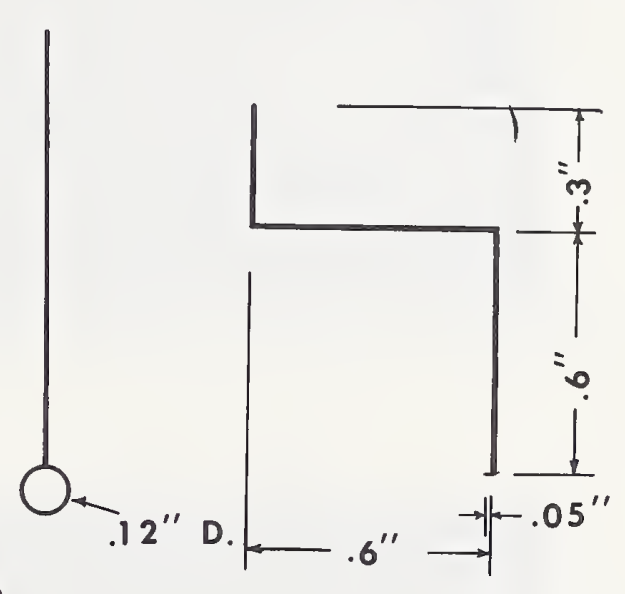

Type B
FLAT FACE

Figure 4.-Flight mill harnesses for codling moth.

The main frame is made of steel and is fastened near both ends for rigidity. Leveling screws and a leveling bulb are used to level the frame. Each sensor is fastened to the steel frame by a compression spring and screws to adjust the distance between the flight arm disk and the sensor for the desired signal sensitivity. The sensor cable is held in position by plastic clips to the main frame. To provide additional protection from damage, the hanging metal straps near each end of the frame can be placed on each side of the frame to hold the flight arms in line with the frame when the unit is not in use. Detailed drawings of the flight mill may be obtained from the senior author.

\section{ADJUSTMENTS ON FLIGHT MILL}

Each flight arm may be adjusted to operate in a horizontal plane by positioning the bottom bearing. This bearing is easily moved because magnetic pull holds it in place on the main steel frame. Each flight arm must be adjusted separately to move in a horizontal plane or it will always come to rest at the lowest point. This can give erroneous instrument readings because the flight arm rotates backward at times before coming to rest and also creates additional drag at each flight initiation. The flight arm should be attached to the bearings so there is always a slight clearance between the pivot pin and bottom bearing. Excessive pressure on the jewel bearings will break them and the friction will cause undesirable drag. To minimize damage to insects from handling, adult moths are attached to the flight arm harness while immobilized in a $40^{\circ} \mathrm{F}$. refrigerated room.

The type A harness (fig. 4) is attached to a moth by dipping the wire loop in Royalbond waterproof contact cement. The loop is then quickly inserted over the abdomen of the moth up to the juncture of the metathorax and 


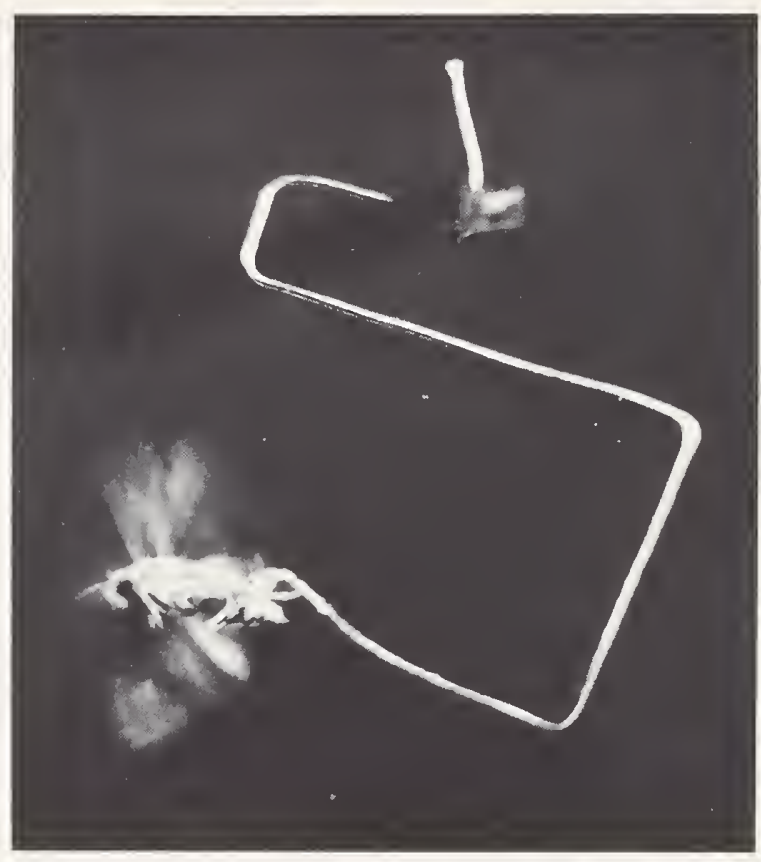

Figure 5.-Codling moth in flight with looped end of harness glued to abdomen.

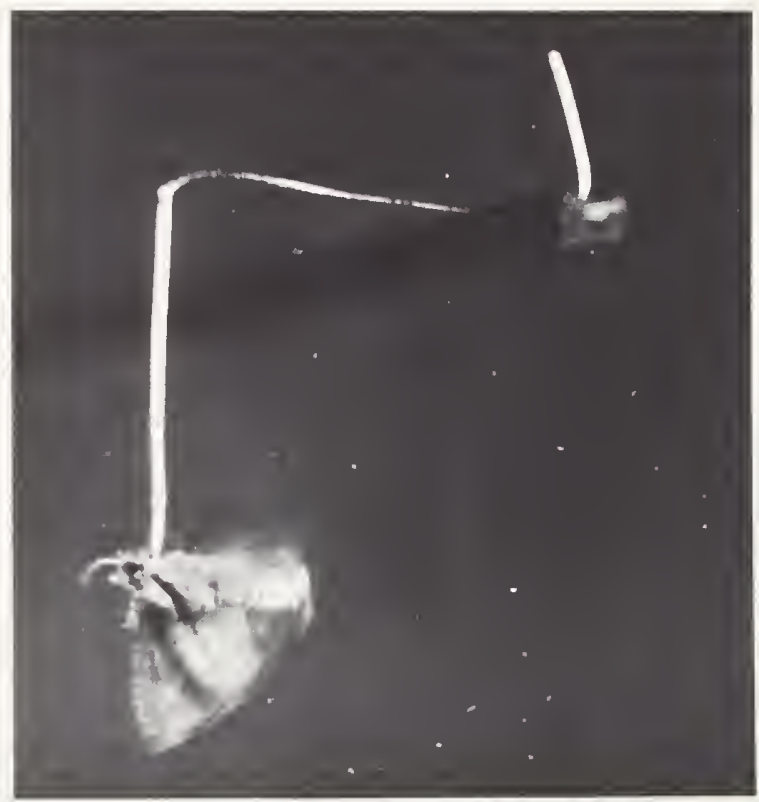

Figure 6.-Codling moth in flight, with flattened wire end of harness glued to pronotum.

first abdominal segment so that the straight portion of the wire leads posteriorly along the dorsum of the moth (fig. 5). A moth can also be successfully harnessed by gluing the flattened end of the wire (type B harness, fig. 4) to the moth's pronotum (fig. 6). These methods of attachment assure a firm connection to the insect without contact with the legs or interference with the wings during flight. The insect harness is attached to the flight mill by passing the free end of the wire through the eye at the end of the flight arm. The insect harness is attached to the flight arm to swing freely in the radial direction, but permits an attached moth to fly in only one circular direction. 
A low-cost flight arm that is simple to construct may be used instead of the jewel-magnet bearing and flight arm unit (fig. 2). The flight arm is made of balsa wood $1 / 32$ by $1 / 16$ inch and the balsa wood is glued to the magnet as shown in fig. 2 . The wood is 25 inches long and weighs 1.25 grams. The magnet weighs 5.75 grams.

A floating magnet is used as a bearing. Two round magnets are mounted with like poles facing each other so that they will repel each other. Each magnet is $3 / 16$ inch thick and $3 / 4$ inch in diameter, with a hole in the center. Magnets made of barium ferrite were selected for uniform magnetic flux density. Each magnet is fitted with a Teflon plug $1 / 4$ inch in diameter and $1 / 4$ inch long. The plug has a hole $1 / 16$ inch in diameter through the center. A brass rod on a block of wood is used to aline the magnets. If desired, the rod may be covered with Teflon to reduce friction. The bearing is fitted loosely for easy rotation. A metal wire harness that holds the insect is attached to the balsa wood at a 12.53-inch radius from the center post. The insect then travels 2 meters per revolution of the flight arm. A sensing disk is glued to the flight arm at the opposite end from the insect to balance the flight arm and to actuate the recording equipment. The sensing disk is shown glued in a vertical position to the flight arm but may be fastened in other positions, depending on requirements of sensing instruments. Sensing components of the type used for the flight mill shown in figure 1 can also be used with the floating-magnet flight arm unit.

Multiple flight arm units mounted on a rod should be separated so that they are not influenced by the magnetism of the adjoining magnets. A small cylindrical rubber (such as a pencil rubber) with a hole drilled in the center can be used to space the units. The rubber is placed on the rod to support the lower magnet of a paired floating-magnet unit.

\section{PERFORMANCE AND USE OF FLIGHT MILLS}

The frictional drag of the floating-magnet flight arm unit is slightly greater than that of the jewel-magnet flight arm unit. However, the codling moths had no difficulty overcoming bearing friction on either flight arm unit. A flight speed of 2 meters in 5 seconds was common. The moths often flew almost continuously for as long as 15 minutes when first at tached to the flight arm. Early tests showed that distances flown by moths during their lives on a flight mill vary considerably. A maximum of 17 miles has been recorded.

This flight mill performed satisfactorily in measuring codling moth flight activity for comparing insect vigor.

A photoelectric cell and light source used for the sensing unit are not recommended where light will affect flight behavior.

If the insect is not alined and attached to the harness properly, its flight will be impaired. 


UNITED STATES DEPARTMENT OF AGRICULTURE Agricultural Research Service Beltsville, Maryland 20705

Official Business

Penaltv for Private Use, $\$ 300$

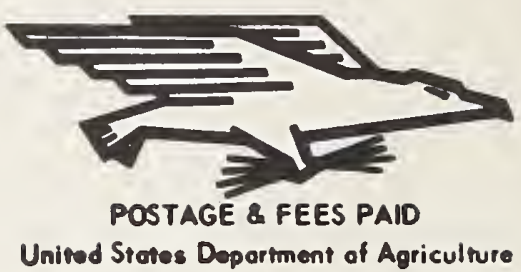

